\title{
MULTIPLE EXPOSITION TO A DRIVING SIMULATOR REDUCES SIMULATOR SYMPTOMS FOR ELDERLY DRIVERS
}

\author{
Normand Teasdale ${ }^{1}$, Martin Lavallière ${ }^{1}$, Mathieu Tremblay ${ }^{1}$, \\ Denis Laurendeau ${ }^{2}$ and Martin Simoneau ${ }^{1}$ \\ ${ }^{1}$ Unité de recherche sur le vieillissement \\ Centre de recherche FRSQ du CHA de Québec \\ ${ }^{2}$ Université Laval \\ Department of Electrical and Computer Engineering \\ Québec, Québec, Canada \\ Email : Normand.Teasdale@kin.msp.ulaval.ca
}

\begin{abstract}
Summary: This study examines how older drivers responded to repeated exposures to a driver simulator. Older active and fit drivers participated in 5 simulator sessions within a 14-day period. For each session, simulator sickness symptoms were measured with the Simulator Sickness Questionnaire at baseline and post-session. In addition, participants completed a $10-\mathrm{cm}$ visual analog scale $(0=$ no symptom, $10=$ mild nausea) at baseline and after a familiarization scenario and post-session. Overall, older adults adapted to the driving simulator and by the fourth session, they showed no difference in sickness scores between the baseline and the post-session measurements. Increasing the exposure duration at session 5 yielded an increase in the sickness symptoms. These results suggest that shorterduration multiple exposures could reduce simulator sickness symptoms in elderly drivers and allow a more effective use of simulators for training by preventing early withdrawal of participants.
\end{abstract}

\section{INTRODUCTION}

Simulator sickness (SS) is often considered a limit precluding widespread use of simulators. According to the most common theory, SS (and motion sickness) is a response to a conflict between received and expected information (Bles, Bos, \& Kruit, 2000; Reason \& Brand, 1975). Presumably, visual-vestibular interactions are at the heart of this conflict. An important corollary of this conflict is that characteristics of a simulator can be a key factor in causing visually induced SS. For instance, Bos et al (2008) reported that a motion mismatch, that is, the difference between the time histories of simulator motion and vehicle motion could explain SS. For most end-users of commercially available driving simulators, control of these particular characteristics is lacking. A better understanding of how subjects adapt to a given driving simulator, however, may help in controlling sickness symptoms that may affect negatively adherence to simulator training programs. Sickness-producing situations are characterized by their lack of familiarity to the participant (Stoffregen \& Riccio, 1988). Most people can adapt to a new motion environment (Johnson, 2005; Reason \& Brand, 1975). Perhaps, one of the most comprehensive study on adaptation is that by Kennedy et al. (2000) who reported both the separate effects of repeated exposures and duration of exposure (within a 3-hour session) on the score obtained at the Kennedy Simulator Sickness Questionnaire (SSQ). Data were obtained from a large data set of helicopter flights from naval pilots $(n=938$ sessions). Exposure duration and repeated exposures were linearly related to sickness outcomes. As the duration of exposure increased the total SSQ score increased. Repeated exposures yielded a gradual decrease in total 
SSQ score. Similar observations have been made elsewhere (Gower, Lilienthal, Kennedy, \& Fowlkes, 1987; Kennedy, Stanney, \& Jones, 1996; Regan, 1995). Similar results for older drivers are scarce. The aim of this study consisted of examining simulator sickness symptoms in a group of older subjects that participated in a multiple-session study aiming at validating simulator training. Although, it has been suggested that elderly are least susceptible to SS than younger adults (Bos, Damala, Lewis, Ganguly, \& Turan, 2007), SS can still be a problem with older participants particularly in studies using fixed-base driving simulators. We thus wanted to examine their adaptation across five sessions. It was expected that elderly would show a decrease in the sickness symptoms with repeated exposure. Second, we also examined if steering control could be a factor in causing SS. This could be the case if small course deviations are detected (visually) too late and overcorrected, leading to an increasing slalom or weaving effect that may aggravate sickness symptoms (Bertin, Collet, Espié, \& Graf, 2005). If this is the case, we should see a decrease in the frequency of steering maneuvers with repeated exposure.

\section{METHODS}

\section{Participants}

22 older participants (age range 65 to 84 years old) participated on voluntary basis. The first aim of the study was to examine the effect of simulator training upon on-road performance. For this purpose, subjects were exposed to 5 simulator sessions on separate days and two on-road sessions that preceded and followed the simulator sessions. Training data are presented in a companion paper. All participants were active drivers with a good general health condition. Simple clinical tests were used to screen for impairments that might affect driving and cognition. Participants also completed a Motion History Questionnaire (MHQ) (Kennedy, Fowlkes, Berbaum, \& Lilienthal, 1992) for previous history of motion sickness.

\section{Procedures}

Simulator. A fixed-based open-cab simulator powered by STISIM Drive 2.0 (Allen, et al., 1997) was used. The simulator consists of an instrumented mid-sized sedan (brake and accelerator pedals, steering and all manual controls) with automatic transmission interfaced with a programmable software (STISIM Drive 2.0 by Systems Technology Inc.) allowing to develop driving scenarios and to record the driver's performance. A projector (Hitachi CP-X275) displays the visual information on a flat wall ( $1.45 \mathrm{~m}$ high $\times 2.0 \mathrm{~m}$ wide) located $2.2 \mathrm{~m}$ from the steering. The center of the screen is located at eye level through the midline of the subject and it allows $40^{\circ}$ horizontal by $30^{\circ}$ vertical field-of-view. Blue curtains on each side of the screen shielded the visual peripheral information. Before the first session, subjects were made aware the simulator could make them feel uncomfortable. They were specifically instructed to inform the experimenter if this happened and were told the experiment would stop immediately without any prejudice for them. The average temperature within the room was $19.5^{\circ} \mathrm{C}(\mathrm{SD}=0.6)$ and ventilation was provided with a ceiling vent positioned just above the driver.

SS measurements. Subjects participated in 5 simulator sessions within 14 days. Before each session (baseline), participants were asked if they were in their usual state of fitness (e.g., not suffering from a cold or flu, hangover, etc.) and first completed the Simulator Sickness 
Questionnaire (SSQ; 16 symptoms rated on a 4-point scale : $0=$ absent, $1=$ slight, $2=$ moderate, $3=$ severe). (Kennedy, Lane, Berbaum, \& Lilienthal, 1993) The ratings form the basis for three subscale scores - Nausea, Oculomotor Discomfort, and Disorientation - as well as a Total severity score. Participants also completed a $10-\mathrm{cm}$ visual analog scale (VAS; $0=$ no symptom, $10=$ mild nausea). Then, a $6-\mathrm{km}$ practice run (with less graphical information than the experimental scenario) served the purpose of familiarizing subjects with the simulator. They filled a VAS score immediately after this practice (post-familiarization) and a 5-min rest was provided. The experimental run followed. For the first and last session, it consisted of a continuous 26-km scenario of urban and rural roads with almost no slopes and minor grade changes. To comply with the 40 degree field of view limitation of our simulator, there was no right or left-turn maneuvers at intersections. Also, moderate curves only were presented (smallest radius of $120 \mathrm{~m}$ ). The scenario included a normal driving environment (two-way and four-way roads, intersections with stop sign or crossing light). Lane width and markings were according to governmental rules and speed limit and advisory signs appeared throughout the scenario. Subjects were asked to follow speed limits and to comply with local traffic laws throughout the course of the experiment. The scenario did not require any emergency braking response unless a driving error was made by the driver. Session 2 to 4 were training sessions with specific feedback on the performance. These sessions varied in length from subject to subject but were shorter in length than session 1 and 5 (about $16 \mathrm{~km}$ vs. $26 \mathrm{~km}$ ). After each of the 5 sessions, subjects completed again the SSQ and a VAS (post-session).

Steering control. To document steering control we looked specifically at overtaking maneuvers embedded into the continuous scenario of the first and last session. Specifically, the drivers were instructed to drive in the rightmost lane except when a recorded message indicated in advance to the driver that he would encounter a slower vehicle and that he should safely overtake the vehicle. In these contexts, drivers needed to adjust their speed with that of the slower vehicle and execute a maneuver requiring to switch lane and move back to the rightmost lane. Seven such maneuvers were presented during the first and last session. We documented the number of steering movements exceeding $2 \mathrm{deg}$. A minimum of three steering movements was needed to complete each maneuver.

\section{RESULTS}

One participant withdrew after the initial session and two participants completed only the first and last session. Data presented herein are from the remaining 19 participants. All participants completed the program in less than two weeks. On average, the time interval between each simulator sessions was 2.4 days $(\mathrm{SD}=1.7)$. Answers to the MHQ revealed that none of the participants used virtual reality equipment. Only one participant reported previous exposure to a driving simulator (one session, two years previous to the actual study). No medication to prevent motion sickness was taken by participants prior to the simulator sessions and none of the participants reported long lasting simulator sickness symptoms.

Figure 1 presents the SSQ total score (left panel) and the VAS (right panel) for each of the five sessions. Data for the three subscales of the SSQ and the total SSQ score were submitted to repeated measures ANOVAs (5 Sessions x 2 Measures; baseline, post-session). All ANOVAs yielded similar results and for sake of brevity, data for the total score only are presented. The 
main effects of Session and Measures $(\mathrm{F}(4,16)=15.25, \mathrm{p}<0.001$ and $\mathrm{F}(1,16)=18.3, \mathrm{p}<0.001$, respectively) and the interaction of Session $x$ Measures $(F(4,16)=12.07, \mathrm{p}<0.001)$ were all statistically significant. All scores were similar at baseline (on average, 8.7). Following the simulations (post-session), we observed a large score after Session 1(on average, 66.7) and this score was different than all other scores. Participants showed a gradual decrease in the SSQ score from the first to the fourth session. By session 4, there was no difference between the baseline and the post-session scores $(\mathrm{p}>0.05)$. Interestingly, however, session 5 which lasted longer than session 2, 3 and 4, yielded an increase in the post-session total score and this post-session score was greater than that observed for the baseline $(\mathrm{p}<0.01)$.
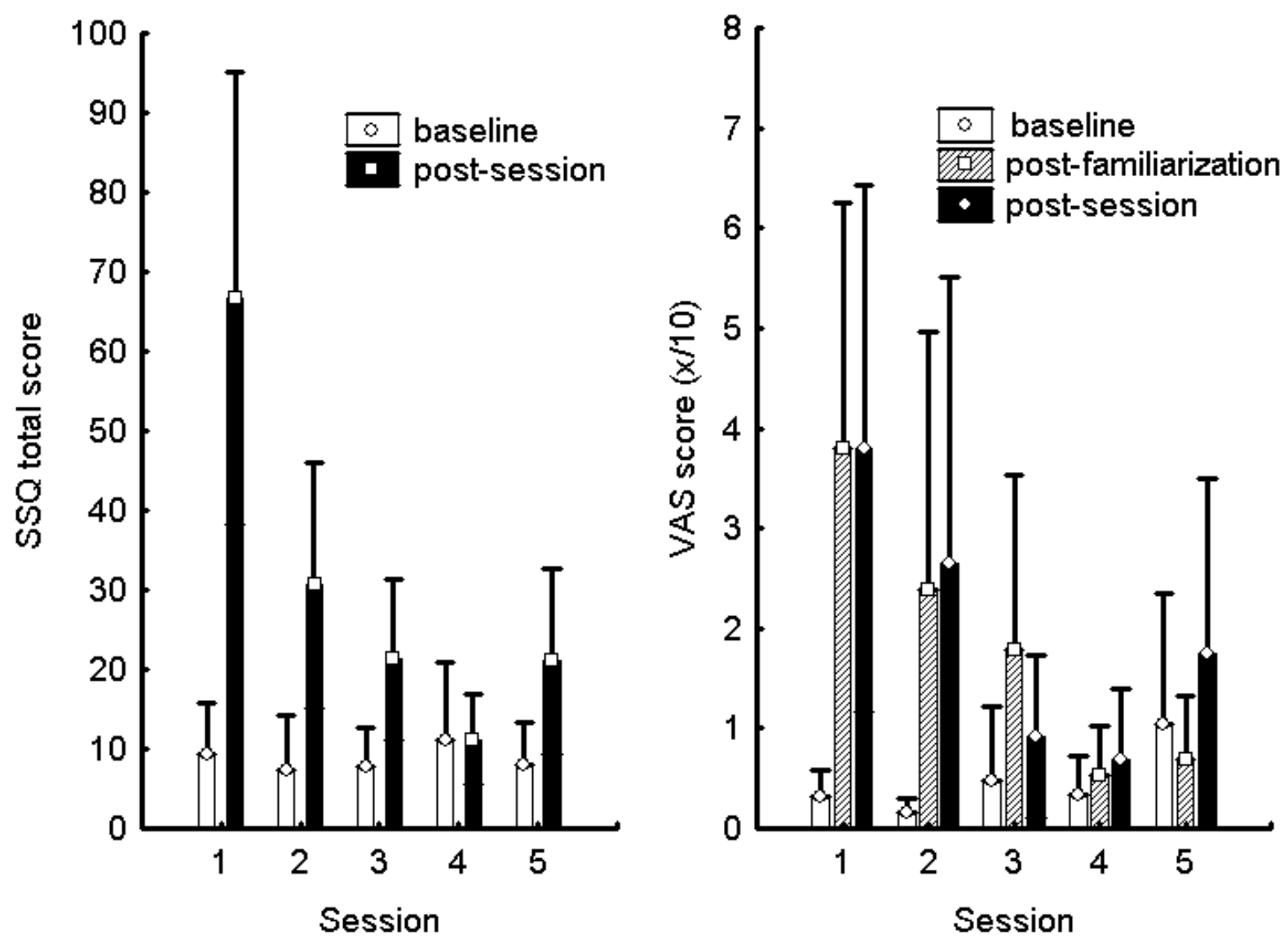

Figure 1. Scores for the Simulator Sickness Questionnaire (SSQ; left panel) and the Visual Analog Scale (VAS; right panel) for the 5 sessions. Error bars indicate the between-subjects $95 \%$ confidence interval.

Because of its ease of use and rapidity to complete, we also collected an intermediate score for the VAS after the familiarization session. Data for the VAS were submitted to a repeated measures ANOVA (5 Sessions x 3 Measures; baseline, post-familiarization, post-session). Overall, similar results to that observed for the SSQ score were obtained and the main effects of Session and Measure $(\mathrm{F}(4,16)=6.04, \mathrm{p}<0.001$ and $\mathrm{F}(2,16)=6.62, \mathrm{p}<0.01$, respectively) and the interaction of Session $x$ Measure $(F(8,16)=4.63, p<0.001)$ were all statistically significant. The baseline VAS was low and similar for all sessions (on average, 0.46). As for the SSQ score, we observed a gradual decrease from the first to the fourth session. It is noteworthy that for the 
first 4 sessions, the VAS after the familiarization was nearly as large as that observed after the experimental run (post-session); none of the comparisons between post-familiarization and postsession showed a significant statistical difference (ps $>0.05$ ). For session 5 , the increase in VAS compared to the baseline score just failed to reach significance $p=0.06$ ). Similarly, the difference between the post-session and the post-familiarization VAS score just failed to reach significance $(\mathrm{p}=0.06)$.

Steering maneuvers were not affected by exposition. On average, participants needed 4.5 steering movements to complete the overtaking maneuvers and there was no difference between the first and the last session ( $\mathrm{p}>0.05)$. This suggests that steering control and additional weaving was not related to higher levels of discomfort for the subjects that completed the study. Although we did not analyze data from subjects that did not complete the study, Rizzo et al. (2003) found no evidence of steering control problems in drivers that withdrew from their study before the point of drop out. Bertin et al. (2005), however, reported more weaving behaviors in sick subjects for 2 out of 3 experiments they conducted.

\section{CONCLUSIONS}

Our data obtained for older drivers parallel those reported by Kennedy et al. (2000) for pilots. Sickness symptoms decreased with multiple exposures. This was confirmed with the SSQ total score (as well as for all sub-scales of the SSQ, not reported in this manuscript) and the VAS score. By the fourth session, no difference was observed for the SSQ and VAS scores obtained at baseline and post-session. However, this is not to say that participants had completely adapted. Indeed, session 5 which was about 10-km longer than session 4 showed that participants, again reported greater symptoms post-session than at baseline.

Effects of exposure duration have been previously reported for a single driving simulator session. For instance, Min et al. (2004) reported that, for young individuals, all SSQ sub-scale scores showed a gradual and monotonic increase every 5 min during a 60 -min testing period. Similarly, Park et al. (2006) reported that young and older individuals showed increasing SSQ scores with exposition time. In these experiments, subjects were exposed to progressively more demanding scenarios. Data from Min et al. (2004), however, suggests that the gradual increase of symptoms in Park et al. (2006) may not be the result of the increased in complexity but mainly of the longer duration of the exposition. The VAS scores observed in our study also support this suggestion. Indeed, our familiarization scenario was mostly linear with few curves, light traffic and few accelerations and decelerations. Yet, for the first three sessions, the score after this familiarization scenario were as high as those obtained after the longer and more complex experimental scenario. Compared to the gradual increase reported by Min et al. (2004) for younger individuals this may suggest a more rapid increase of sickness symptoms and increased sensitivity for older individuals. By session 4, post-familiarization and post-session SSQ and VAS scores were not different than baseline scores. At session 5, increasing the exposure duration, however, yielded an increase in sickness symptoms suggesting that exposure duration is an important factor to consider. Multiple exposures of short-durations could lessen simulator sickness and favor effective simulator training for older drivers that are more susceptible to SS. Such strategies could allow to decrease the number of dropouts in driving simulator studies 


\section{ACKNOWLEDGEMENTS}

Special thanks to all drivers that participated in the project. This project (COBVIS-D) received support from AUTO21 (www.auto21.ca) and the Société de l'Assurance Automobile du Québec (SAAQ).

\section{REFERENCES}

Allen, R. W., Rosenthal, T. J., Aponso, B. L., Klyde, D. H., Anderson, F. G., Hogue, J. R., et al. (1997). A Low Cost PC Based Driving Simulator for Prototyping and Hardware-In-TheLoop Applications, SAE International Congress \& Exposition.

Bertin, R. J. V., Collet, C., Espié, S., \& Graf, W. (2005). Objective measurement of simulator sickness and the role of visual-vestibular conflict situations. Paper presented at the DSC 2005, Orlando.

Bles, W., Bos, J. E., \& Kruit, H. (2000). Motion sickness. Current Opinion in Neurology, 13(1), 19-25.

Bos, J. E., Bles, W., \& Groen, E. L. (2008). A theory on visually induced motion sickness. Displays, 29, 47-57.

Bos, J. E., Damala, D., Lewis, C., Ganguly, A., \& Turan, O. (2007). Susceptibility to seasickness. Ergonomics, 50(6), 890-901.

Gower, D. W., Lilienthal, M. G., Kennedy, R. S., \& Fowlkes, J. E. (1987). Simulator sickness in U.S. Army and Navy fixed-and rotary-wing flight simulators. Paper presented at the AGARD Medical Panel Symposium in Motion Cues in Flight Simulation and Simulator Induced Sickness, Brussels, Belgium.

Johnson, D. M. (2005). Introduction to and review of simulator sickness research (No. 1832): U.S. Army Research Institute for the Behavioral and Social Sciences.

Kennedy, R. S., Fowlkes, J. E., Berbaum, K. S., \& Lilienthal, M. G. (1992). Use of a motion sickness history questionnaire for prediction of simulator sickness. Aviat Space Environ Med, 63(7), 588-593.

Kennedy, R. S., Lane, N. E., Berbaum, K. S., \& Lilienthal, M. G. (1993). Simulator Sickness Questionnaire: An Enhanced Method for Quantifying Simulator Sickness. The International Journal of Aviation Psychology, 3(3), 203-220.

Kennedy, R. S., Stanney, K. M., \& Dunlap, W. P. (2000). Duration and Exposure to Virtual Environments: Sickness Curves During and Across Sessions. Presence, 9(5), 463-472.

Kennedy, R. S., Stanney, K. M., \& Jones, M. B. (1996). Simulator sickness questionnaire: Normative data and exposure guidelines. Paper presented at the 1996 Annual Scientific Meeting of the Aerospace Medical Association., Atlanta, GA.

Min, B. C., Chung, S. C., Min, Y. K., \& Sakamoto, K. (2004). Psychophysiological evaluation of simulator sickness evoked by a graphic simulator. Applied Ergonomics, 35(6), 549-556.

Park, G. D., Allen, R. W., Fiorentino, D., Rosenthal, T. J., \& Cook, M. L. (2006). Simulator Sickness Scores According to Symptom Susceptibility, Age, and Gender for an Older Driver 
Assessment Study. Paper presented at the Human Factors and Ergonomics Society 50th Annual Meeting, San Fransisco, CA.

Reason, J. T., \& Brand, J. J. (1975). Motion sickness. London ; New York: Academic Press.

Regan, C. (1995). An investigation into nausea and other side-effects of head-coupled immersive virtual reality Virtual Reality, 1(1), 17-31.

Rizzo, M., Sheffield, R. A., Stierman, L., \& Dawson, J. (2003). Demographic and driving performance factors in simulator adaptation syndrome. Paper presented at the Second International Driving Symposium on Human Factors in Driver Assessment, Training and Vehicle Design, DesignPark City, Utah.

Stoffregen, T. A., \& Riccio, G. E. (1988). An ecological theory of orientation and the vestibular system. Psychological Review, 95(1), 3-14. 